\title{
Interaction of Blood Lead and 8-Aminolevulinic Acid Dehydratase Genotype on Markers of Heme Synthesis and Sperm Production in Lead Smelter Workers
}

\author{
Bruce H. Alexander, ${ }^{1}$ Harvey Checkoway, ${ }^{1,2}$ Paola Costa-Mallen, ${ }^{1}$ Elaine M. Faustman, ${ }^{1,2,3,4}$ James S. Woods, ${ }^{1,2}$ \\ Karl T. Kelsey, ${ }^{5}$ Chris van Netten, ${ }^{6}$ and Lucio G. Costa ${ }^{2}$
}

${ }^{1}$ Department of Environmental Health, ${ }^{2}$ NIEHS Center for Ecogenetics and Environmental Health, ${ }^{3}$ Institute for Risk Analysis and Risk Communication, and ${ }^{4}$ Center on Human Development and Disability, School of Public Health and Community Medicine, University of Washington, Seattle, WA 98195 USA; ${ }^{5}$ Occupational Health Program, Department of Environmental Health and Department of Cancer Biology, Harvard School of Public Health, Boston, MA 02115 USA; ${ }^{6}$ Department of Health Care and Epidemiology, University of British Columbia, Vancouver, BC V6T 123 Canada

The gene that encodes $\delta$-aminolevulinic acid dehydratase (ALAD) has a polymorphism that may modify lead toxicokinetics and ultimately influence individual susceptibility to lead poisoning. To evaluate the effect of the ALAD polymorphism on lead-mediated outcomes, a cross-sectional study of male employees from a lead-zinc smelter compared associations between blood lead concentration and markers of heme synthesis and semen quality with respect to ALAD genotype. Male employees were recruited via postal questionnaire to donate blood and urine for analysis of blood lead, zinc protoporphyrin (ZPP), urinary coproporphyrin (CPU), and ALAD genotype, and semen samples for semen analysis. Of the 134 workers who had ALAD genotypes completed, $114(85 \%)$ were ALAD1-1 (ALAD1) and 20 (15\%) were ALAD1-2 (ALAD2). The mean blood lead concentrations for ALAD1 and ALAD2 were 23.1 and $28.4 \mu \mathrm{g} / \mathrm{dl}(p=0.08)$, respectively. ZPP/heme ratios were higher in ALAD1 workers ( 68.6 vs. $57.8 \mu \mathrm{mol} / \mathrm{mol} ; p=0.14$ ), and the slope of the blood lead ZPP linear relationship was greater for ALAD1 (2.83 vs. 1.50, $p=$ 0.06). No linear relationship between CPU and blood lead concentration was observed for either ALAD1 or ALAD2. The associations of blood lead concentration with ZPP, CPU, sperm count, and sperm concentration were more evident in workers with the ALAD1 genotype and blood lead concentrations $\geq 40 \mu \mathrm{g} / \mathrm{dl}$. The ALAD genetic polymorphism appears to modify the association between blood lead concentration and ZPP. However, consistent modification of effects were not found for CPU, sperm count, or sperm concentration. Key words: $\delta$-aminolevulinic acid dehydratase, coproporphyrin, genotype, lead, semen, smelters, zinc protoporphyrin. Environ Health Perspect 106:213-216 (1998). [Online 12 March 1998]

http://ehpnet1.niehs.nih.gov/docs/1998/106p213-216alexander/abstract.html

Lead is recognized as a cause of secondary porphyria resulting from heme synthesis inhibition, characterized by elevated levels of blood $\delta$-aminolevulinic acid (ALA) and zinc protoporphyrin (ZPP) and urinary ALA and coproporphyrin (CPU) (1). The biological effects of lead can be detected at blood lead concentrations below current U.S. occupational health protection standards, which require medical removal in cases of repeated blood lead concentrations exceeding $50 \mu \mathrm{g} / \mathrm{dl}$ or one blood lead concentration exceeding $60 \mu \mathrm{g} / \mathrm{dl}$ (2). Biological markers of heme synthesis inhibition can reveal the effects of lead at various stages in the heme synthesis pathway. Lead inhibits the second enzyme in the pathway, aminolevulinic acid dehydratase (ALAD), which synthesizes porphobilinogen from ALA (3). Decreased ALAD activity results in an increase of free ALA, the presence of which is postulated as one mechanism for the neurotoxic effects of lead (4). The action of lead at this point in the heme synthesis pathway is estimated by measuring the activity of ALAD in blood or the concentration of ALA in blood or urine. The formation of protoporphyrinogen IX from coproporphyrinogen III is inhibited by lead, resulting in an increase in CPU. Inactivation of coproporphyrinogen oxidase or impaired transport of coproporphyrinogen into mitochondria may underlie this effect (5). Lead also interferes with the chelation of iron into protoporphyrin IX to form heme. Whether the mechanism is due to ferrochetalase inhibition is unclear (๑). Instead of heme, zinc protoporphyrin (ZPP) is formed. ZPP is considered a sensitive marker of lead exposure and is a commonly used marker of leadinduced heme synthesis inhibition.

The determinants of lead kinetics and individual susceptibility to lead toxicity in humans are not clearly defined, despite considerable experimental animal research. Interindividual variation in the effects of lead exposure is probably governed by a complex web of susceptibility that includes the type and route of exposure, nutritional status, gender, age, and genetic profile. A possible genetic component of variation in the bodily response to lead exposure is the gene that encodes ALAD.
ALAD is encoded by a single gene with two common alleles, ALAD1 and ALAD2, which are expressed as three distinct phenotypes: ALAD1-1, ALAD1-2, and ALAD2-2 (7). ALAD2, the less common variant, is prevalent in $10-20 \%$ of the Caucasian population $(7-11)$. This polymorphism appears to modify the toxicokinetics of lead $(12,13)$, and the presence of the ALAD2 allele has been associated with higher blood lead levels in lead-exposed workers and children $(11,14)$. There are conflicting findings as to whether ALAD2 carriers are more susceptible to the ultimate health effects of environmental lead exposure. The ALAD2 genotype putatively enhances red cell binding of lead, which may increase blood lead burden and thus be viewed as harmful (15). Alternatively, the ALAD2 genotype may be associated with reduced lead deposition in other body compartments and may thus protect against systemic toxicity including impaired heme synthesis (12). This study describes the interaction of the effects of lead and the presence of the ALAD polymorphism on markers of heme synthesis and reproductive health in a population of male lead smelter workers.

\section{Methods}

This cross-sectional study was part of a larger study on the effects of occupational lead exposure on male reproductive health conducted at a lead-zinc smelter in Trail, British Columbia (16). Male employees of the smelter $(n=2,469)$ were recruited by postal questionnaire to participate in the study. Volunteers were solicited to donate

Address correspondence to B.H. Alexander, Department of Environmental Health, Box 358772, University of Washington, Seattle, WA 98195 USA. This research was sponsored by National Institutes of Environmental Health Sciences grants ES04696 and ES07033, National Institutes of Health grants ES005947 and ES00002, National Institute for Occupational Safety and Health grant R03$\mathrm{OH} 02966$, and Department of Energy grant DEFC01-95EW. Bruce H. Alexander and Paola CostaMallen were supported by NIEHS training grant T32-ES07262.

Received 14 July 1997; accepted 17 November 1997. 
semen, blood, and urine samples. The study protocol was approved by the University of Washington Human Subjects Committee, and informed consent for study participation was obtained from the volunteers. Venous blood samples were drawn by the company occupational health nurse into EDTA-containing tubes. The samples were refrigerated at $4^{\circ} \mathrm{C}$ until analyzed for blood lead and ZPP. Blood lead concentrations were measured using graphite furnace atomic absorption spectrophotometry (17). ZPP levels were ascertained by hematofluorometry (18) and reported as $\mathrm{ZPP} /$ heme ratios; residual blood samples were then stored at $-20^{\circ} \mathrm{C}$. ALAD genotype was determined for each worker for which a residual blood sample was available. Genotyping for ALAD was performed by the polymerase chain reaction method of Wetmur ( $\nearrow$ ) with protocol modifications described by Smith et al. (13).

Spot morning-void urine samples were collected by the workers and delivered to the laboratory the day of or the day after the blood was drawn and were stored at $-20^{\circ} \mathrm{C}$ until analyzed. Porphyrin profiles were assessed by HPLC-spectrofluorometric procedures (19). CPU was expressed as a concentration by volume (micrograms per liter) and by weight (micrograms per gram creatinine).

Semen samples were collected at home or on-site after a requested 48 -hr period of abstinence from sexual activity and delivered to the field laboratory within $1 \mathrm{hr}$ of collection. A complete semen analysis was performed according to the World Health Organization protocol (20). The results for sperm concentration and total sperm count, previously reported to be associated with blood lead concentration in this population (16), are presented here.

Data were initially explored using correlation analyses and scatter plots. Mean blood lead, ZPP/heme ratio, CPU, sperm count, and sperm concentration were compared by ALAD genotype. The relationships between blood lead concentration and markers of heme synthesis inhibition (ZPP and CPU) and sperm count and concentration were evaluated using multiple linear regression with separate models for each $A L A D$ genotype and both genotypes combined (21). The slopes of the blood lead ZPP associations for the two genotypes were compared in a model with an interaction term for blood lead concentration and ALAD genotype. Age adjustment was performed in all models.

An additional analysis was conducted in reference to blood lead levels at or above regulatory action levels. Mean ZPP and $\mathrm{CPU}$ and geometric means of sperm count and sperm concentration were compared by genotype and blood lead concentration less than and greater than or equal to 40 $\mu \mathrm{g} / \mathrm{dl}$. This cutpoint was established based on an Occupational Safety and Health Administration worker health protection criterion that requires additional monitoring of any worker with a blood lead concentration $>40 \mu \mathrm{g} / \mathrm{dl}$. Removal from work is not required until the worker has repeated measures $>50 \mu \mathrm{g} / \mathrm{dl}$ or a single measurement $\geq 60 \mu \mathrm{g} / \mathrm{dl}(2)$.

\section{Results}

Blood samples were donated by 152 workers (16), among whom there was sufficient residual sample to determine ALAD genotype for 134 workers. Porphyrin profiles could be determined for 119 of the genotyped workers. One hundred fourteen $(85 \%)$ of the 134 workers were homozygous for the ALAD type 1 (ALAD1-1) and 20 (15\%) were heterozygous (ALAD 1-2) (Table 1). No workers were homozygous for the type 2 allele. Compared to workers with ALAD2, workers with ALAD1 were slightly older (mean age 40.1 vs. 39.0 years) and had slightly longer average tenure (17.5 vs. 15.8 years), but fewer years in the higher lead exposure areas of the smelter ( 4.3 vs. 4.7). Semen samples were available for 106 workers among those for whom ALAD genotyping was completed: 89 were ALAD1 and 17 were ALAD2. The mean blood lead concentration of workers with ALAD2 was greater than those with ALAD1 (28.4 vs. $23.1 \mu \mathrm{g} / \mathrm{dl}, p=0.08)$. In contrast, the $\mathrm{ZPP} /$ heme ratios were lower for workers with the ALAD2 genotype (57.8 vs. 68.6 $\mu \mathrm{mol} / \mathrm{mol}, p=0.14)$. Differences in geometric mean sperm count and concentration were minimal.

The linear associations between blood lead, ZPP, and ALAD genotype are displayed in Table 2 and Figures 1 and 2. As expected, blood lead concentration was strongly predictive of ZPP $\left(R^{2}=0.47\right)$. The relationship between blood lead and ZPP appeared to be modified by ALAD genotype. The slope of this relationship for ALAD1 workers was $2.83(p<0.0001)$, compared to $1.50(p=0.0004)$ for ALAD2 workers. The coefficient for the interaction of ALAD genotype and blood lead level on ZPP was $1.30(p=0.06)$. Urinary coproporphyrin excretion did not vary by blood lead concentration on a linear scale for either genotype ( $R^{2}<0.03$ for all models). The log values of total sperm count and sperm concentration were inversely correlated with blood lead concentration ( $p=$ 0.02 and 0.10 , respectively), but this association did not vary by $A L A D$ genotype.

Differences for ZPP and CPU levels by ALAD genotype were evident when workers with blood lead levels below and greater than or equal to $40 \mu \mathrm{g} / \mathrm{dl}$ were compared (Table 3). These differences were more pronounced at or above $40 \mu \mathrm{g} / \mathrm{dl}$. Mean CPU concentration for ALAD1-1 was

Table 1. Distribution of age, blood lead concentration, indicators of heme synthesis, and sperm count and concentration by $\delta$-aminolevulinic acid dehydratase (ALAD) genotype in lead smelter workers

\begin{tabular}{lcccc}
\hline & ALAD1-1 $(n=114)$ & & ALAD1-2 $(n=20)$ & \\
\cline { 2 - 2 } \cline { 5 - 5 } & Mean \pm SD & & Mean \pm SD & $p$-Value \\
\hline Age & $40.1 \pm 7.1$ & & $39.0 \pm 7.8$ & 0.58 \\
Blood lead $(\mu \mathrm{g} / \mathrm{dl})$ & $23.1 \pm 12.2$ & & $28.4 \pm 11.7$ & 0.08 \\
ZPP $(\mu \mathrm{mol} / \mathrm{mol}$ heme) & $68.6 \pm 48.0$ & & $57.8 \pm 24.4$ & 0.14 \\
Coproporphyrin & $47.4 \pm 41.6$ & & $41.6 \pm 36.8$ & 0.59 \\
Creatinine-adjusted coproporphyrin & $20.8 \pm 19.5$ & & $19.3 \pm 15.7$ & 0.76 \\
Sperm concentration & $60.3 \pm 3.3^{\mathrm{a}}$ & & $60.3 \pm 2.1^{\mathrm{a}}$ & 0.96 \\
Total sperm count & $134 \pm 3.7^{\mathrm{a}}$ & & $148 \pm 2.5^{\mathrm{a}}$ & 0.91 \\
\hline
\end{tabular}

SD, standard deviation.

aGeometric mean and geometric SD.

Table 2. Linear regression models of zinc protoporphyrin regressed on blood lead concentration by $\delta$ aminolevulinic acid dehydratase (ALAD)

\begin{tabular}{|c|c|c|c|c|c|}
\hline Model & $n$ & Variables $^{a}$ & $\beta$ & $p$-Value & $R^{2}$ \\
\hline Combined & 134 & $\begin{array}{l}\text { Blood lead } \\
\text { Age }\end{array}$ & $\begin{array}{l}2.53 \\
0.76\end{array}$ & $\begin{array}{c}<0.0001 \\
0.07\end{array}$ & 0.47 \\
\hline ALAD1-1 & 114 & $\begin{array}{l}\text { Blood lead } \\
\text { Age }\end{array}$ & $\begin{array}{l}2.83 \\
0.96\end{array}$ & $\begin{array}{c}<0.0001 \\
0.04\end{array}$ & 0.52 \\
\hline ALAD1-2 & 20 & $\begin{array}{l}\text { Blood lead } \\
\text { Age }\end{array}$ & $\begin{array}{r}1.50 \\
-0.74\end{array}$ & $\begin{array}{l}0.0004 \\
0.18\end{array}$ & 0.57 \\
\hline Interaction & 134 & $\begin{array}{l}\text { Blood lead } \\
\text { Age } \\
\text { ALAD } \\
\text { ALAD*Blood lead }\end{array}$ & $\begin{array}{r}1.53 \\
0.68 \\
-11.59 \\
1.30\end{array}$ & $\begin{array}{l}0.02 \\
0.09 \\
0.58 \\
0.06\end{array}$ & 0.52 \\
\hline
\end{tabular}

Variable coding: ALAD: ALAD1 = 1, ALAD2 = 0; blood lead concentration $=\mu \mathrm{g} / \mathrm{dl} ;$ zinc protoporphyrin $=\mu \mathrm{mol} / \mathrm{mol}$ heme; age $=$ years. 


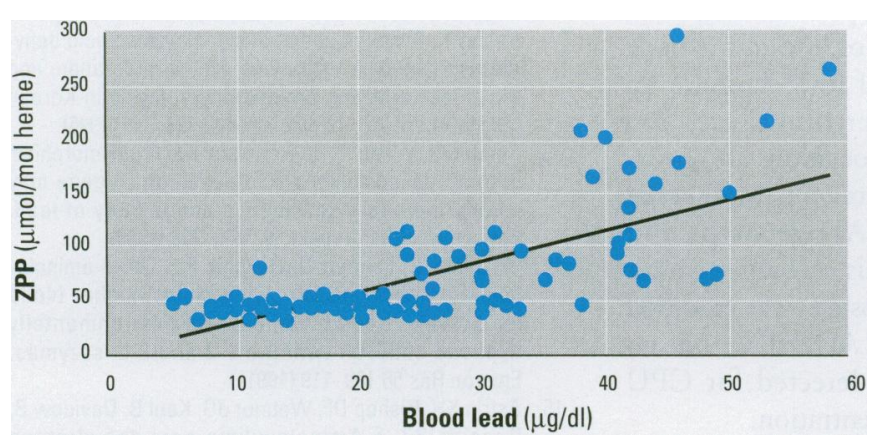

Figure 1. Zinc protoporphyrin (ZPP) level by current blood lead concentration for workers with ALAD1-1 genotype.

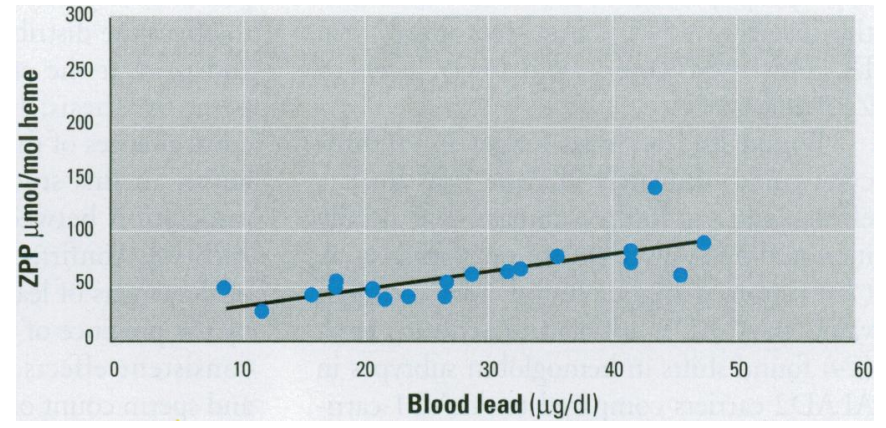

Figure 2. Zinc protoporphyrin (ZPP) level by current blood lead concentration for workers with ALAD1-2 genotype. threefold greater than for ALAD1-2 (46.5 vs. $13.6 \mu \mathrm{g} / \mathrm{l})$. A similar association, albeit attenuated, was seen for the creatinineadjusted CPU. CPU did not, however, increase with increasing blood lead concentration as would be expected. The effect of blood lead concentrations $>40 \mu \mathrm{g} / \mathrm{dl}$ on total sperm count and sperm concentration were most apparent in workers with the ALAD type 1 genotype. The geometric mean sperm count and concentration values for ALAD1 workers in this blood lead range were approximately half those with ALAD type 2 genotype. These results, however, are not statistically significant, as they are based on only four workers with ALAD2 and a blood lead concentration $>40 \mu \mathrm{g} / \mathrm{dl}$.

\section{Discussion}

The association of blood lead concentration and ZPP was modified by ALAD genotype in this population of male lead smelter workers. Despite having lower blood lead concentrations, workers with the ALAD1 genotype had, on average, higher ZPP levels compared to those with ALAD2. This difference was most pronounced at blood lead levels $>40 \mu \mathrm{g} / \mathrm{dl}$.

The nonrandom method of worker recruitment, the study sample size, and the absence of female workers were the primary limitations of this cross-sectional study. The smelter workers volunteered to participate by donating a blood sample following recruitment via a mailed questionnaire. The participation rate was low, in large part due to the semen sample donation requirement of the original study; nevertheless, the volunteers were similar to the overall population with respect to age, length of employment, work area, and blood-lead monitoring history (16). It is unlikely that ALAD genotype played a role in a worker's decision to participate. The prevalence of the ALAD1-2 allele was in the expected range of $10-20 \%(7-11)$. The small number of workers with the variant

Table 3. Zinc protoporphyrin, coproporphyrin, sperm count, and sperm concentration by $\delta$-aminolevulinic acid dehydratase (ALAD) genotype and blood lead concentration above and below $40 \mu \mathrm{g} / \mathrm{dl}$

\begin{tabular}{|c|c|c|c|c|c|c|}
\hline & \multirow[b]{2}{*}{ Blood lead $(\mu \mathrm{g} / \mathrm{dl})$} & \multicolumn{2}{|c|}{ ALAD1-1 } & \multicolumn{2}{|c|}{ ALAD1-2 } & \multirow[b]{2}{*}{$p$-Value } \\
\hline & & $n$ & Mean \pm SD & $n$ & Mean \pm SD & \\
\hline Zinc protoporphyrin & $<40$ & 98 & $55.7 \pm 27.1$ & 15 & $48.5 \pm 12.5$ & 0.10 \\
\hline ( $\mu \mathrm{mol} / \mathrm{mol}$ heme) & $\geq 40$ & 16 & $147.3 \pm 70.3$ & 5 & $85.6 \pm 31.5$ & 0.03 \\
\hline Coproporphyrin & $<40$ & 90 & $47.5 \pm 42.9$ & 13 & $50.3 \pm 38.1$ & 0.81 \\
\hline$(\mu \mathrm{g} / \mathrm{l})$ & $\geq 40$ & 12 & $46.5 \pm 31.6$ & 4 & $13.6 \pm 7.8$ & 0.01 \\
\hline Creatinine-adjusted & $<40$ & 90 & $21.2 \pm 20.1$ & 13 & $22.9 \pm 15.9$ & 0.76 \\
\hline coproporphyrin $(\mu \mathrm{g} / \mathrm{g})$ & $\geq 40$ & 12 & $18.4 \pm 14.0$ & 4 & $7.5 \pm 7.2$ & 0.16 \\
\hline Sperm concentration & $<40$ & 82 & $63.6(2.9)^{a}$ & 13 & $61.1(2.2)^{a}$ & 0.89 \\
\hline (million sperm/ml) & $\geq 40$ & 7 & $32.2(7.4)^{a}$ & 4 & $61.8(1.9)^{a}$ & 0.54 \\
\hline Total sperm count & $<40$ & 82 & $152(3.4)^{a}$ & 13 & $155(3.4)^{a}$ & 0.95 \\
\hline (million sperm) & $\geq 40$ & 7 & $58(10.0)^{a}$ & 4 & $116(3.0)^{a}$ & 0.59 \\
\hline
\end{tabular}

$\mathrm{SD}$, standard deviation.

${ }^{a}$ Geometric mean (geometric SD).

allele represented a sparse distribution of blood lead concentrations, with few exceeding $40 \mu \mathrm{g} / \mathrm{dl}$. The statistical power of the study to model the exposure-gene interaction was limited accordingly. The absence of female workers limits the generalizability of these data particularly insofar as gender may modify the effects of lead on heme synthesis (22).

The ALAD polymorphism appears to influence the toxicokinetics of lead. However, the evidence of either allele predisposing an individual to the adverse health effects of lead is inconclusive. In the current study mean blood lead levels of workers with the ALAD2 genotype were somewhat higher than those with ALAD1. Similar observations have been made of occupationally exposed adults and environmentally exposed children $(11,12,14)$. Despite the higher blood lead concentrations, the association between blood lead and ZPP levels of ALAD2 workers was attenuated. This phenomenon was also reported in a study of Korean storage-battery-manufacturing workers where a subgroup of workers with the highest lead exposures had lower ZPP concentrations when the ALAD2 genotype was present (12).

Suggestive of a similar mechanism, $\mathrm{CPU}$ concentrations were lower in workers with the ALAD2 allele when blood lead levels exceeded $40 \mu \mathrm{g} / \mathrm{dl}$. However, this observation may be an artifact because the overall CPU concentration was not associated with blood lead concentration. Intraindividual variation of urinary coproporphyrin concentration, especially measured in spot samples, can be large (1), which limits its utility as a biomarker of heme synthesis toxicity. The semen quality parameters of total sperm count and sperm concentration were also suggestive of a possible protective effect of ALAD2 genotype for workers with blood lead levels $\geq 40$ $\mu \mathrm{g} / \mathrm{dl}$. Although these differences were based on too few individuals to be statistically stable, they do contribute evidence that the ALAD polymorphism may modify the health effects of lead.

A mechanism for the modified associations between blood lead and ZPP by ALAD genotype has not been clearly established. It has been hypothesized that the variant form of ALAD (ALAD2) may bind lead more tightly than ALAD1 and make it less bioavailable (23). Should this be the case, given constant exposure, less lead would be bioavailable for inhibition of heme synthesis downstream of ALAD in this biochemical pathway. The result of this enhanced protein binding of lead in ALAD2 carriers would be a decrease in the amount of bioavailable lead to inactivate 
coproporphyrinogen oxidase or to inhibit the insertion of iron into protoporphyrin IX. This then would yield lower levels of ZPP and CPU.

Bound lead retained longer in erythrocytes could ultimately increase risks for late effects, such as kidney damage and mildly increased hemoglobin turnover. Smith et al. (13) reported higher blood urea nitrogen values in ALAD2 carriers and Schwartz et al. (24) found shifts in hemoglobin subtypes in ALAD2 carriers compared to ALAD1 carriers. This theory is also supported by apparent differential partitioning by ALAD genotype of lead in bone of carpenters with low blood lead levels (13). The theoretical differential binding of lead by the ALAD isozymes does not indicate greater susceptibility to lead for persons with the ALAD2 genotype. In the current study, apparent inhibition of heme synthesis (elevated ZPP) was more pronounced in workers with the ALAD1 genotype, suggesting that they, rather than the workers with ALAD2 and greater blood lead concentrations, are predisposed to the adverse hematologic effects of lead. The neurological effects of lead may be similarly altered. An elevated concentration of plasma ALA was shown to be higher in battery workers with homozygous ALAD1 genotype compared to heterozygous ALAD 1-2 workers (25). This effect was independent of blood lead concentration and age. To the extent that the increased ALA concentration contributes to the neurological effects of lead, persons with homozygous ALA1 genotype may be more susceptible to the neurological effects of lead. Though based on few cases, adolescents with the ALAD2 genotype scored higher than those with ALAD1 on a battery of neuropsychological tests (26). Conversely, ALAD2 carriers with low blood lead concentrations showed more evidence of impaired renal function (13).

The role of the ALAD genetic polymorphism in conferring susceptibility to the adverse health outcomes associated with lead exposure has yet to be clearly defined. The presence of the ALAD type 2 allele appears to affect the distribution of lead in the body and to alter the effect of lead exposure on heme synthesis; however, the causes and consequences of these associations are speculative. In this study a previously observed association between ALAD genotype and ZPP was confirmed. This sensitive marker of the effects of lead exposure was modified by the presence of the ALAD2 allele, but no consistent effects were detected for CPU and sperm count or concentration.

\section{RefERENCES}

1. Daniell WE, Stockbridge HL, Labbe RF, Woods JS, Anderson KE, Bissell DM, Bloomer JR, Ellefson RD, Moore MR, Pierach CA, Schreiber WE, Tefferi A, Franklin GM. Environmental chemical exposures and disturbances of heme synthesis. Environ Health Perspect 105(suppl 1):37-53 (1997).

2. Occupational Safety and Health Standards. Sub-part Z: Toxic and Hazardous Substances-Lead. 29 CFR 1910.1025. Washington, DC:Office of the Federal Register, 1985.

3. Bernard A, Lauwerys R. Metal-induced alterations of $\delta$-aminolevulinic acid dehydratase. Ann NY Acad Sci 514:41-47 (1987).

4. Moore MR, Goldberg A, Yeung-Laiwah AA. Lead effects on the heme biosynthetic pathway: relationship to toxicity. Ann NY Acad Sci 514:191-203 (1987).

5. Woods JS. Porphyrin metabolism as indicator of metal exposure and toxicity. In: Handbook of Experimental Pharmacology, Vol 115. Toxicology of Metals-Biochemical Aspects (Goyer RA, Cherian MG, eds). Berlin:Springer-Verlag, 1995;19-52.

6. Labbé RF, Rettmer RL. Zinc protoporphyrin: a product of iron deficient erythropoiesis. Semin Hematol 26:40-46 (1989).

7. Wetmur JG, Kaya AH, Plewinska M, Desnick RJ. Molecular characterization of the human $\delta$-aminolevulinate dehydratase 2 (ALAD2) allele: implications for molecular screening of individuals for genetic susceptibility to lead poisoning. Am J Hum Genet 49:757-763 (1991)

8. Battistuzzi G, Petrucci R, Silvagni L, Urbani FR, Caiola S. $\delta$-Aminolevulinate dehydrase: a new genetic polymorphism in man. Ann Hum Genet 45:223-229 (1981).

9. Petrucci R, Leonardi A, Battistuzzi G. The genetic polymorphism of $D$-aminolevulinate dehydrase in Italy. Hum Genet 60:289-290 (1982).

10. Benkmann HG, Bogdanski P, Goedde HW. Polymorphism of delta-aminolevulinic acid dehydratase in various populations. Hum Hered 33:62-64 (1983).

11. Ziemsen B, Angerer J. Lehnert G, Benkmann HG Goedde HW. Polymorphism of delta-aminolevulinic acid dehydratase in lead exposed workers. Int Arch Occup Environ Health 58:245-247 (1986).
12. Schwart BS, Lee BK, Stewart W, Ahn KD, Springer K Kelsey K. Associations of $\delta$-aminolevulinic acid dehydratase genotype with plant, exposure duration, and blood lead and zinc protoporphyrin levels in Korean lead workers. Am J Epidemiol 142:738-745 (1995).

13. Smith CM, Wang $X$, Hu H, Kelsey KT. A polymorphism in the $\delta$-aminolevulinic acid dehydratase gene may modify the pharmacokinetics and toxicity of lead. Environ Health Perspect 103:248-253 (1995).

14. Wetmur JG, Lehnert G, Desnick RJ. The $\delta$-aminolevulinate dehydratase polymorphism: higher blood lead levels in lead workers and environmentally exposed children with the 1-2 and 2-2 isozymes. Environ Res 56:109-119 (1991).

15. Astrin KH, Bishop DF, Wetmur JG, Kaul B, Davidow B, Desnick RJ. $\delta$-Aminolevulinic acid dehydratase isozymes and lead toxicity. Ann NY Acad Sci 514:23-29 (1987).

16. Alexander BH, Checkoway $H$, van Netten C, Muller CH, Ewers TH, Kaufman JD, Mueller BA, Vaughan TL, Faustman EM. Semen quality of men employed at a lead smelter. Occup Environ Med 53:411-416 (1996).

17. Henry JB. Clinical Diagnosis and Management by Laboratory Methods, $16^{\text {th }}$ ed. Philadelphia, PA:WB Saunders Co., 1979;513-515.

18. Rettmer RL, Labbé RF. Zinc protoporphyrin/heme ratio: evaluation of the Proto Fluor System. Clin Chem 2:1194 (1986).

19. Woods JS, Bowers MA, Davis HA. Urinary porphyrin profiles as biomarkers of trace metal exposure and toxicity: studies on urinary porphyrin excretion patterns in rats during prolonged exposure to methyl mercury. Toxicol Appl Pharmacol 110:464-476 (1991).

20. World Health Organization. WHO Laboratory Manual for the Examination of Human Semen and SemenCervical Mucus Interaction. Cambridge, UK:Cambridge University Press, 1992.

21. Kleinbaum DG. Kupper LL, Muller KE. Applied regression analysis and other multivariable methods. 2nd ed. Boston:PWS-Kent, 1988.

22. Oishi $H$, Nomiyama $H$, Nomiyama K, Tomokuni $K$. Comparisons between males and females with respect to the porphyrin metabolic disorders found in workers occupationally exposed to lead. Int Arch Occup Environ Health 68:298-304 (1996).

23. Wetmur JG. Influence of the common human deltaaminolevulinate dehydratase polymorphism on lead body burden. Environ Health Perspect 102(suppl 3):215-219 (1994).

24. Schwart BS, Lee BK, Stewart W, Ahn KD, Kelsey K, Bressler J. Associations of subtypes of hemoglobin with delta-aminolevulinic acid dehydratase genotype and dimercaptosuccinic acid-chelatable lead levels. Arch Environ Health 52:97-103 (1997).

25. Sithisarankul P, Schwartz BS, Lee BK, Kelsey KT, Strickland PT. Aminolevulinic acid dehydratase genotype medicates plasma levels of the neurotoxin, 5 aminolevulinic acid, in lead-exposed workers. Am J Ind Med 32:15-20 (1997).

26. Bellinger D, Hu H, Titlebaum L, Needleman HL. Attentional correlates of dentin and bone lead levels in adolescents. Arch Environ Health 49:98-105 (1994). 